\title{
XXXl. Neue Arten aus: H. Mayr, Fremdländische Wald- und Parkbäume für Europa. 1906.
}

\section{Larix Cajanderii Mayr, 1. c., p. 297. (Cajanders Lärche.)}

Die neue Lärche bildet von der Mïndung des Aldan in die Lerıa (63 Grad n. Br.) an nordwärts Mischbestände mit der sibirischen Fichte (Picea obovata) oder mit Betula odorata; reine Lärchenbestände mit über $20 \mathrm{~m}$ Höhe finden sich nur auf Böden, welche nicht mehr überschwemmt werden: von der Aldanmündung südwärts scheint alles $L$. dahurica zu sein, was von Lärchen sich zeigt; auf Sandboden wächst die europäische Föhre Pinus silvestris; auf der Meeresinsel Tit Ary unter dem 72. Grad wird Cajanders Lärche nur noch $3-6 \mathrm{~m}$ hoch.

Cajanders Lärche ist folgendermassen gekennzeichnet: Junge Kurztriebnadeln haben anfangs Juni noch nicht ihre normale Grösse erreicht; an zapfentragenden Zweigen verbliebene, vorjährige Nadeln besassen die ungewöhnliche Länge von 4-5 cm. Beim Platzen der Knospen erscheint mit den neuen Nadeln ein dichter, weissgelber, lockiger Haarschopf, welcher der dahurica feh]t, wohl aber der Prinz-Rupprechts-Lärche ebenfalls zukommt. Einjährige Triebe hellgelb-braun mit einzelnstehenden Haaren besetzt; mehrjährige 'Triebe hellaschfarbig; Zapfen kurz, 1-1,5 cm lang, mit ca. 20 Schuppen; diese am oberen Rand ausgebuchtet, nicht gekerbt wie dahurica; wenn trocken, klaffen die Schuppen wio bei dahurica und kurilensis. Zapfenspindel behaart; Zapfenstiel haarlos.

Ostsibirien.

2. Larix Principis Rupprechtii Mayr, I. c., p. 309. (Prinz-RupprechtsLärche.)

Einjährige fertige Triebe hellgelb an den herablaufenden Nadelbasen mit zwischenliegenden mattgraugrünen Tönen, kahl, soweit der Larix europaea sehr ähnlich; an den Seitentrieben Nadeln sichelförmig gekrümmt; Knospe der Kurztriebe mit zahlreichen hellgelben, langen Haaren innerhalb der Schuppen, Knospenfarbe rotbraun. Hierin somit sehr verschieden von $L$. sibirica. Zaplen an einem kräftigen, aufwärts gekrümmten, $2 \mathrm{~cm}$ langen Stiele sitzend; Zapfen $4 \mathrm{~cm}$ lang und $3 \mathrm{~cm}$ Durchmesser, wenn geöffnet. An schwächlichen Exemplaren sind die Zapfen selbstverständlich, wie bei allen Nadelhölzern, kleiner und deshalb für die Systematik unbrauchbar. Zapfenschuppen nach dem öffnen des Zapfens ganz flachgedrückt, fein gezähnt, kahl; Blütenschuppen nur an der Basis zwischen den Zapfenschuppen hervortretend, von der Mitte des Zapfens an aufwärts unsichtbar. Die rasch erwachsenden Lärchen zeigen in ihrer Heimat völlig geraden Schaftbau; im übrigen in Tracht Bau von Rinde und Borke der europäischen Lärche ähnlich; auch das Holz ist an durchschnittenen Stämmen in Splint und Kern von gleicher äuBerer Beschaffenheit, wie bei der europäischen Lärche des kühleren Verbreitungsgebietes beobachtet wird. 
Die Lärche habe ich zu Ehren Seiner Königlichen Hoheit des Prinzen Rupprecht von Bayern benannt.

Wutajshan, Nordchina.

3. Picea Mastersil Mayr, l. c., p. 328. (Mastersfichte.)

Einjähriger, fertiger Trieb hell ockerfarbig, zweijähriger rahmweiss; Knospe stumpf, hellockerfarbig, etwas glänzend; Nadeln der jungen Pflanze scharf stechend, vierkantig im Querschnitte; mit weissen Spaltöffnungsstreifen auf allen Seiten. Nadeln des fruchttragenden Baumes in Grösse ausserordentlich wechselnd von $2-10 \mathrm{~mm}$, und in fast rechtem Winkel vom Zweige abstehend. Zapfen an einem kurzen Stiele, der am Zweige verbleibt; Zapfenbasis selbst gegen die Anhaftstelle hin konisch zulaufend. Zapfenlänge stets nur sehr wenig schwankend, von $5--6 \mathrm{~cm}$ und $3 \mathrm{~cm}$ Durchmesser, wenn offen. Zapfen hellgelbbraun, schwach glänzend; Schuppen abgerundet, mit schwacher Kerbe am obersten Rande; dieser Teil stets nach der Zapfenspindel hin gekrümmt; die Blütenschuppe trägt zwei deutlich hervorragende Harzgänge. Die Rinde des erwachsenen Baumes ist kleinschuppig, hellgrau; die Tracht eines erwachsenen, freistehenden Baumes erinnert an eine Tanne.

Wutaishan, China.

4. Picea Tschonoskii Mayr, I. c., p. 339. (Tschonoskisfichte.)

Eine sehr bekannte, bisher unter dem Namen Picea Maximovicsii = Picea obovata japonica verbreitete Art. Mit kurzen, scharf stechenden Nadeln, fast rechtwinklig vom Triebe abstehend; Triebfarbo hellrotbraun $m$ ersten Jahre; im zwoiten Jahre mit hellgelbgrauen Längslinien zwischen den rotbraunen Resten des ersten Jahres. Knospe braun mit bläulichem Schimmer. Anscheinend nur ein Halbbaum. Zapfen der AlbaFichte täuschend ähnlich. Wegen der Wuchsverhältnisse hat man sie den Hochalpen von Japan zugeschrieben. Masters vermutet mit Recht. dah ihre Heimat wahrscheinlich China sein werde. Am interessantesten ist an dieser Fichte, dass unter den obigen beiden Namen von den Gärtnern und Pflanzenhandlungen eine ganz andere Fichte vertrieben wird, als Regel und Maximovics darunter verstanden haben. Entscheidend hierüber ist folgender Fund: Im Herbarium zu Kew fand ich eines der Originalexemplare, welche Tschonoski 1864 , in Japonia, Fujiyama" sammelte, und welche im Ind. sem. hort. Petro. von Maximovics als Picea obovata var. japonica, von Regel später als Picea Maximovicsii benant wurde. Das von Tschonoski am Fuji-noyama gesammelte Exemplar ist nun ganz zweifellos Picea bicolor in ihrer normalen, typischen Form. Picea obovata var. japonica Max. und Picea Maximovicsii Regel sind daher in ihrer ursprünglichen Beschreibung und Bedeutung nichts als Nadelsynonyme der systematisch solange von Systematikern und besonders von Gärtnern arg misshandelten Spezies Picea bicolor; damit ist die als Picea Maximovicsii von den Gärtnern kultivierte Art ohne Namen; ich habe ihr den Namen dessen gegeben, der den Samen der fraglichen Holzart, unbekannt woher, nach Petersburg sandte. 
Heimat unbekannt, wahrscheinlich China, sicher nicht Japan.

5. Betula wutaica Mayr, J. c., p. 450. (Wutaibirke.)

Blätter kurzgestielt, fast herzförmig, aber mit gerade abgeschnittenem Grunde, gezähnt; Rippen unterseits mit Borstenhaaren, ebenso Blattstiel behaart. Rinde der jungen Triebe braun, kahl, warzig; am dreijährigen Triebe platzt die Rinde in rotgelbe, dünne Papierborke auf. Fruchtzäpfchen aufrechtstehend, zylindrisch, Spindel kahl: Zapfenschuppen dreiteilig, stark gebuckelt; im Winkel jeder Schuppe 2-3 Samen; Same an den Narbenresten Borsten tragend. Am älteren Baume erscheint frühzeitig eine grobrindige, schwarze Borke.

Wutaishan, China.

6. Populus wutaica Mayr, l. c., p. 494. (Wutaipappel.)

Diese zur Gruppe der Zitterpappeln gehörige Art bewohnt das im Nordwesten von Peking gelegene Wutaigebirge und die westlichen Berge, wo ich sie an den Südhängen unter den Resten der ehemaligen Laubwaldvegetation fand. Blatt des erwachsenen Baumes fast kreisrund, nur unterster Rand grob wellig gekerbt, oben in eine Spitze endend; Ausschlagblätter sehr grob, derb, mit groben, stumpfen Zähnen; alles kahl, wenn erwachsen; wenn jung, weibfilzig behaart. Blattstiel plattgedrückt. Knospen am Triebe anliegend, Schuppen mit dunkelbraunem Rand, ebenfalls glatt; Deckschuppe der Blüte hellockerfarbig, am Rande mit weissen Seidenhaaren; Fruchtknoten kahl. Kätzchenspindel kahl; Rinde an jungen Stämmen hellgrau-grün mit rhombischen Lentizellen.

Wutaishan, China.

7. Quercus wutaishanica Mayr, l. c., p. 504. (Wutaischaneiche.)

Blatt gelappt; grösste Blattbreite im oberen Drittel: Rippen, Blattstiele und junge Triebe borstig behaart, von den Trieben bald abfallend; Knospen klein, am Grunde mit fadenartigen, bewimperten Blättchen, die frühzeitig absterben und während des Winters die Knospe überragen. Rand der Knospenschuppen behaart. Eichel klein, Becher mit kurzwolligen Schüppchen. Rinde des erwachsenen Baumes eine graue, kleinschuppige Borke. Vorstehende Eiche steht der mongolischen Eiche (Quercus mongolica Fisch.) sehr nahe.

Wutaiberge, China.

\section{Vermischte neue Diagnosen.}

402. Forsythia Giraldiana Lingelsheim in Jahrb. Schles. Ges. Vaterl. Kultur, LXXXVI, 1908 (1909), II b, p. 1.

Frutex. Gemrnae fuscae, glabrae; perulae margine tenuissime ciliatae. Rami erecti, subquadrangulati, grisei, lenticellis sparsis obtecti; ramuli ochracei, saepius falcato-curvati. Folia indivisia, 6-10 $\mathrm{cm}$ longa, 2,5$5 \mathrm{~cm}$ lata, subcoriacea, ambitu oblonga vel ovalia, basin versus sensim attenuata, apicem versus subcaudato-acuminata, margine integerrima, glaberrima vel secus nervos leviter pilosa, petiolo $0,5-1 \mathrm{~cm}$ longo in- 